\title{
Distribución de polimorfismos asociados al grado de infiltración de grasa intramuscular en siete razas bovinas de carne utilizadas en la Región de La Araucanía, Chile ${ }^{\#}$
}

\author{
Polymorphism distribution associated with marbling degree in seven beef cattle breeds being \\ present in La Araucanía Region, Chile \\ J Piñeira*, J Río, H Floody, R Felmer \\ Laboratorio de Biotecnología Animal, Instituto de Investigaciones Agropecuarias (INIA), Carillanca, Temuco, Chile.
}

\begin{abstract}
SUMMARY
Selection of breeding stock is a crucial step in any breeding program, therefore the methodology for animal evaluation has evolved rapidly and in recent decades has incorporated the use of molecular markers. Several multinational companies have made available to producers commercial tests based on these technologies, which allow assessing the predisposition of animals to express a specific phenotype. However, some doubts have begun to arise about negative effects of improper use of molecular markers, these are problems associated with inbreeding depression and long-term loss of response to selection. Therefore, the objective of this study was to assess allele and genotype frequencies of 4 polymorphisms associated with marbling in 7 beef cattle breeds being present in La Araucanía Region of Chile. We evaluated leptin, thyroglobulin, DGAT1 and FABP4 genes, which showed different frequency distributions among breeds with higher allele frequency for marbling in Aberdeen Angus breed and a low frequency in Belgian Blue and Hereford breeds. We conclude that it is feasible to use these tools in genetic programs, but it is also necessary to have reliable genealogical records and advice of professional or technical institutions that can integrate genotypic information from molecular markers in prediction models used for selection of breeding stock.
\end{abstract}

Palabras clave: criterio de selección, marcadores moleculares, BLUP.

Key words: selection criteria, molecular markers, BLUP.

\section{INTRODUCCIÓN}

La selección de los reproductores de la siguiente generación es un paso fundamental en todo programa de mejora genética. Para realizar esta selección, el mejorador debe utilizar el valor genético aditivo de los individuos, dejando para reposición aquellos animales cuya combinación de genes es la mejor para el carácter de interés. El objetivo de esta selección es maximizar la ganancia genética por generación mediante un correcto ordenamiento por mérito genético de los candidatos a ser reproductores (Buxade 1995).

La genética ha dedicado un continuo esfuerzo a la obtención, a partir de la información fenotípica de un individuo y la de sus parientes, de un predictor del valor genético que se libere de los efectos ambientales y posea propiedades estadísticas óptimas (Buxade 1995). De esta manera, la metodología para la evaluación de los animales ha evolucionado rápidamente, y en las últimas décadas se ha pasado desde aquellas técnicas que consideraban tan

Aceptado: 14.10.2011.

\# Proyectos Capital Humano Avanzado (ex PBCT) PSD63 e INNOVACORFO 07CN13PBM-06.

* Laboratorio de Biotecnología Animal, Instituto de Investigaciones Agropecuarias, INIA-Carillanca. Camino Cajón Vilcún s/n, km 10, Casilla 58-D, Temuco, Chile; jpineira@inia.cl solo parte de la información genealógica, mediante índices de selección (Smith 1936, Hazel 1943, Henderson 1990), a otras más sofisticadas que emplean toda la información (genealogía, sexo, raza, predio, variables productivas, etc.) mediante la metodología BLUP (Best Linear Unbiased Predictor) propuesta por Henderson (1949).

De forma paralela, durante la segunda mitad del siglo XX los progresos realizados en biología molecular permitieron importantes avances en la identificación de las variaciones genéticas que se producen a nivel de la secuencia del ADN. La actividad ganadera no ha estado al margen de dichos avances y grandes multinacionales como Pfizer, Animal Genetics, Igenity de Merial e Illumina, entre otras, han puesto en el mercado pruebas comerciales que ofrecen la posibilidad de seleccionar reproductores mediante la identificación de polimorfismos asociados a fenotipos de interés productivo como producción de leche, longevidad, fertilidad, calidad de la carcasa, etc. ${ }^{1}$.

Aunque existe consenso en que es poco probable que en un futuro cercano la selección mediante marcadores moleculares logre desplazar el uso de herramientas cuantitativas en programas de mejoramiento genético (Powell y Norman 2006), es claro que dichas herramientas pueden ser utilizadas como un importante criterio de selección (Buxade

www.pfizeranimalgenetics.com.au/default.aspx; http://www.igenity. com 
1995), especialmente para aquellas características de difícil medición o de manifestación tardía, como la cobertura grasa, la terneza o el grado de infiltración grasa.

Este último carácter, también conocido como marmóreo, se refiere al contenido de grasa intramuscular, es decir, a la presencia de franjas o manchas blancas de tejido graso entre las fibras musculares de la carne (Barton-Gade y col 1988). En la actualidad existen estudios que señalan que la heredabilidad del marmóreo fluctúa entre moderada a alta (Harper y Pethick 2001), y que su expresión a nivel fenotípico presenta diferencias significativas entre distintas razas.

Por ejemplo, se ha observado que las razas lecheras (Holstein, Jersey) muestran puntajes más altos de marmóreo que las razas británicas (Angus, Hereford, Shorthorn), las cuales a su vez muestran mejores puntajes que otras razas europeas (Limousin, Simmental, Charolais). Más aún, todas ellas alcanzan mejores puntajes que las razas índicas (cebuinas), independiente de la edad y el tipo de alimentación (Harper y Pethick 2001).

El marmóreo es uno de los principales parámetros utilizados para la valoración de la calidad de la carne ya que influye directamente en sus características organolépticas. Por esta razón, en numerosos países como Canadá se pagan bonificaciones a los ganaderos que produzcan carnes de calidad AAA o Premium (Corpoica 2005). Incentivos como los anteriormente señalados han justificado la realización de diversos estudios destinados a identificar variantes alélicas en genes candidatos que confieran a los animales una mayor predisposición a depositar grasa intramuscular. Entre estos genes es posible mencionar el gen de la leptina, hormona que actúa en el control del peso, regulando el consumo de alimento y el gasto energético (Santos-Alvarez y col 1999, Kadokawa y col 2000, Block y col 2001). Fitzsimmons y col (1998) describieron una asociación entre los niveles de grasa de la canal en bovinos y el microsatélite BM1500, que se ubica 3,6 kb río abajo del codón de detención del gen de la leptina (Stone y col 1996). Estas evidencias sugirieron que la leptina podría ser un buen candidato para evaluar su efecto en las propiedades de las grasas depositadas en la canal del ganado. Más tarde, Buchanan y col (2002) describieron que una transición de citosina $(C)$ a timina (T) en el codón 25 del exón 2 del gen de la leptina (posición 466 de la accesión GenBank AJ512638) cambia el aminoácido arginina por cisteína. Posteriormente, el análisis de un gran número de animales de distintas razas (Buchanan y col 2002, Kononoff y col 2005) llevó a concluir que el alelo $\mathrm{T}$ se asocia con canales grasas y el alelo $\mathrm{C}$ a canales magras.

Otro gen de gran importancia es el de la tiroglobulina, proteína precursora de la tiroxina (hormona que afecta el metabolismo) y que ha sido correlacionada con el contenido de grasa intramuscular. Estudios desarrollados por Barendse (1999) relacionaron un posible QTL en el cromosoma 14 con el microsatélite CSSM34, el que a su vez presentaba un ligamiento muy cercano con el gen de la tiroglobulina. Este antecedente, sumado al hecho de que la tiroglobulina es el precursor de hormonas que afectan el metabolismo de los lípidos, posicionó a este gen como un candidato funcional para estudios de selección asistida por marcadores. Posteriormente se observó que en ganado de las razas Holstein alemana y Charolais, los animales homocigotos TT en la posición 1696 de la accesión de GenBank M35823 de este gen tenían más grasa en el músculo longisimus dorsi que animales heterocigotos CT u homocigotos CC (Thaller y col $2003^{b}$, Casas y col 2007). Este hallazgo fue posteriormente validado por el Consorcio Nacional de Evaluación de Ganado de Carne (NBCEC, del inglés National Beef Cattle Evaluation Consortium), en animales generados a partir de cruzas Simmental x Angus, derivando en una de las primeras pruebas comerciales disponibles para los productores.

Junto a los polimorfismos antes descritos se encuentra una variante alélica de la enzima microsomal diacilglicerol-O-aciltransferasa (DGAT1) que cataliza el paso final de la síntesis de triglicéridos (Buhman y col 2001). Originalmente, Riquet y col (1999) analizaron una serie de características productivas en el desempeño de vacas lecheras, especialmente en el contenido de grasa de la leche y lograron asociar un QTL en el cromosoma 14 bovino, el cual fue identificado por dos grupos distintos como el gen que codifica para DGAT1 (Grisart y col 2002, Winter y col 2002). Estudios posteriores confirmaron la existencia de una mutación en las posiciones 10433 y 10434 (AAG/ GCG) (número de accesión de GenBank AJ318490) que da lugar a la sustitución del aminoácido lisina $\left(\right.$ DGAT $^{K}{ }^{K}$ ) por alanina $\left(\mathrm{DGAT}^{A}\right)$. Esta mutación afecta la actividad de esta enzima, lo que se traduce en un efecto sobre el rendimiento y composición de la leche, observación que ha sido ratificada en diversos estudios (Grisart y col 2002, Spelman y col 2002, Thaller y col 2003a , Grisart y col 2004). Más tarde, Thaller y col $\left(2003^{b}\right)$ extendieron esta observación a la calidad de la carne bovina, informando que el contenido de grasa intramuscular (especialmente en musculus semitendinosus) era mayor en animales homocigotos para el alelo lisina $\left(\mathrm{DGAT} 1^{K}\right.$ ), lo cual incidía directamente en el marmóreo de la carne.

Otra variante alélica de interés para el carácter de infiltración grasa es la descrita para la proteína de unión a ácidos grasos (FABP4, del inglés Fatty acid binding protein), la cual pertenece a una pequeña familia de biomoléculas altamente conservadas que se unen a las proteínas citoplasmáticas, ácidos grasos de cadena larga y otros ligandos hidrófobos que tienen entre otras funciones la absorción de ácidos grasos, el transporte y el metabolismo (Kaikaus y col 1990). Dicha proteína posee una mutación en la posición 7516 (número de accesión de GenBank AAFC01136716) en la que se sustituye una guanina por una citosina, mutación que se encuentra significativamente correlacionada con el nivel de infiltración grasa y cobertura grasa en animales producto de las cruzas de Waygu 
x Limousin (Michal y col 2006, Jiang y Michal 2007). De igual manera, recientemente Barendse y col (2009) correlacionaron positivamente un nuevo polimorfismo ( . $2502 \mathrm{C}>\mathrm{G}$ ) en este gen en la raza Aberdeen Angus, lo que confirma la asociación de al menos dos polimorfismos de FABP4 con el fenotipo de mayor infiltración grasa.

El uso de marcadores moleculares como criterio de selección ha comenzado a utilizarse en muchos países desarrollados y también en países en vías de desarrollo como Chile. Por esta razón, resulta clave comprender que la incorporación de estas herramientas conlleva tanto ventajas como desventajas. Algunas de sus ventajas están en la posibilidad de decidir anticipadamente si debemos conservar o sacrificar a un animal sin esperar a que este manifieste el fenotipo de interés. De igual manera, la incorporación de marcadores moleculares permitiría disminuir el número de candidatos que entren a pruebas de progenie, las cuales resultan costosas para muchos productores, además de la reducción de los intervalos generacionales como resultado de progresos genéticos más rápidos (Shanks 2008). No obstante lo anterior, es necesario tener en cuenta que existen factores que pueden dificultar la aplicación de programas de mejoramiento genético asistidos por marcadores moleculares. Entre estos factores destaca el hecho de que la mayoría de los caracteres de calidad de la carne y la canal están influenciados por muchos genes (poligenes), y por lo tanto el seguimiento de un bajo número de estos a través de marcadores de ADN sólo explicará una pequeña proporción de la varianza genética. Debido a lo anterior, autores como Gordon $(2001)^{2}$ y Kinghorn $(2005)^{3}$ plantean que la selección genética basada únicamente en la selección genómica no será ciento por ciento confiable, hasta que todos los genes mayores y menores hayan sido plenamente identificados y validados.

De igual manera, existe evidencia de que la excesiva e inadecuada presión selectiva generada sobre un número limitado de genes puede llegar a producir problemas de endogamia (Gibson 1994), lo que sumado al hecho de que en países como Chile la mayoría de los criadores no cuentan con registros genealógicos confiables, podría dar lugar a importantes reducciones de variabilidad genética, aparición de enfermedades asociadas a la depresión endogámica y la pérdida de capacidad de respuesta ante eventuales cambios de los objetivos de selección (Shanks 2008).

Conforme lo anterior, el objetivo del presente trabajo fue determinar las frecuencias génicas y genotípicas de cuatro polimorfismos asociados a la infiltración grasa en siete razas bovinas utilizadas para producción de carne en la Región de La Araucanía y discutir sobre el eventual uso de dichos marcadores moleculares en programas de mejoramiento genético.

\footnotetext{
http://beefmagazine.com/mag/beef_silver_bullet_science/index. html

3 http://www2.dpi.qld.gov.au/beef/2244.html
}

\section{MATERIAL Y MÉTODOS}

\section{OBTENCIÓN DE MUESTRAS}

Los análisis se realizaron a partir de muestras obtenidas desde 439 animales distribuidos en siete razas bovinas de carne localizadas en 11 predios de la Región de La Araucanía, Chile: Angus Negro (AN), Angus Rojo (AR), Hereford (HF), Belga Azul (BA), Bazadaise (BD), Clavel de Carne Chileno (CC) y Simmental (SI). Las muestras se tomaron mediante la realización de una biopsia en el pabellón auricular de cada animal, utilizando un alicate y una cápsula especialmente diseñada para tal propósito (sistema biopsytec) ${ }^{4}$. Posteriormente, las muestras fueron trasladadas al Laboratorio de Biotecnología Animal de INIA Carillanca, ubicado a $20 \mathrm{~km}$ de la ciudad de Temuco, donde fueron conservadas en un ultrafreezer a $-80{ }^{\circ} \mathrm{C}$, para su posterior análisis.

\section{EXTRACCIÓN DEL ADN}

En esta etapa se procedió a recuperar el tejido guardado en las cápsulas de biopsia, el que fue lavado cuidadosamente con agua destilada, secado en toallas de papel y puesto en un tubo eppendorf de $1,5 \mathrm{ml}$. Posteriormente se agregaron en cada tubo $500 \mu \mathrm{l}$ de tampón de digestión (Tris-HCl $100 \mathrm{mM} \mathrm{pH} 8,5$, EDTA 5mM, NaCl $200 \mathrm{mM}$, SDS 0,20\% y proteinasa $\mathrm{K} 100 \mu \mathrm{g} / \mathrm{ml}$ ). La mezcla se incubó a $55^{\circ} \mathrm{C}$ toda la noche en una placa térmica con agitación.

$\mathrm{Al}$ otro día se centrifugó por cinco minutos a máxima velocidad para separar las impurezas, y luego se trasladó el sobrenadante a un tubo nuevo, al que se le agregaron $500 \mu \mathrm{l}$ de isopropanol. Dicho tubo se mezcló por inversión 20 veces, lo que dio lugar a la formación de un ovillo de $\mathrm{ADN}$, que se recuperó con una punta de micropipeta y se depositó en un nuevo tubo. Finalmente, el ADN obtenido se lavó con $1 \mathrm{ml}$ de etanol al 70\%, se secó en una estufa a $37^{\circ} \mathrm{C}$, se resuspendió en $100 \mu \mathrm{l}$ de tampón T.E. y se guardó a $-20{ }^{\circ} \mathrm{C}$.

\section{AMPLIFICACIÓN Y GENOTIPADO DE LOS MARCADORES}

Se amplificaron los genes de leptina (LEP), tiroglobulina (TG), diacilglicerol-O-aciltransferasa (DGAT1) y proteína de unión a ácidos grasos 4 (FABP4).

La amplificación del gen de la leptina y la posterior identificación de la mutación $466 \mathrm{C}>\mathrm{T}$ se realizó mediante la técnica ARMS-PCR ("Amplification Refractory Mutation System-Polymerase Chain Reaction"), la cual emplea cuatro partidores, dos de los cuales son específicos para cada alelo del polimorfismo a estudiar (Corva y col 2009). La detección de los polimorfismos de TG, DGAT1 y FABP4 se realizó de acuerdo al método de PCR-RFLP ("Polymerase Chain Reaction-Restriction Fragment Lenght

www.biopsytec.de 
Cuadro 1. Polimorfismos evaluados en los genes de leptina, tiroglobulina, DGAT1 y FABP4 y las secuencias de partidores utilizados en la etapa de amplificación.

Polymorphisms in leptin, thyroglobulin, DGAT1 and FABP4 genes and primer sequences used in amplification stage.

\begin{tabular}{llll}
\hline Locus & Polimorfismo & Accesión GeneBank & \multicolumn{1}{c}{ Partidores } \\
\hline leptina & g.466C $>$ T & AJ512638 & 5'-GAC GAT GTG CCA CGT GTG GTT -3' \\
& & & 5'- CGG TTC TAC CTC GTC TCC CAG-3' \\
& & & 5'-TGT CTT ACG TGG AGG CTG TGC CCA TCT -3' \\
& & 5'- AGG GTT TTG GTG TCA TCC TGG ACC TTT CG-3' \\
Tiroglobulina & g.1696 C $>\mathrm{T}$ & M35823 & 5'-GGG GAT GAC TAT GAG TAT GAC TG-3' \\
& & & 5'-GTG AAA ATC TTG TGG AGG CTG TA-3' \\
DGAT1 & 10433-10434 & AJ318490 & 5'-GCA CCA TCC TCT TCC TCA AG-3' \\
& (AAG/GCG) & & 5'-GGA AGC GCT TTC GGA TG-3' \\
FABP4 & g.7516G $>\mathrm{C}$ & AAFC01136716 & 5'-ATA TAG TCC ATA GGG TGG CAA AGA-3' \\
& & & 5'-AAC CTC TCT TTG AAT TCT CCA TTC T-3' \\
\hline
\end{tabular}

Polymorphism") y las condiciones descritas por Thaller y col $\left(2003^{b}\right)$, Kaupe y col (2004) y Michal y col (2006), respectivamente.

Todas las reacciones de PCR se llevaron a cabo en un termociclador Esco modelo Swift Maxi utilizando los partidores que se presentan en el cuadro 1. Para la separación de los fragmentos de restricción se utilizó electroforesis en geles de agarosa al $2 \%$ en tampón T.B.E. 1x, durante 45 minutos a $94 \mathrm{~mA}$. Para su visualización, los geles fueron teñidos con bromuro de etidio y fotografiados mediante un equipo de fotodocumentación Fujifilm Las-3000.

\section{ANÁLISIS DE DATOS}

El análisis de datos contempló la estimación de las frecuencias génicas y genotípicas observadas y esperadas para cada uno de los loci en las razas evaluadas, además de una prueba de equilibrio de Hardy y Weinberg (Falconer y MacKay 1996). Para tal efecto, se utilizaron los métodos de Weir y Cockerham (1984) y Robertson y Hill (1984) mediante los cuales se estimó el estadístico $F_{I S}$ (Wright 1965). En el proceso de análisis se emplearon los programas GenePop 4.0.10 (Raymond y Rousset 1995), Genetix 4.04 (Belkhir y col 2004) y FSTAT 2.9.3.2. (Goudet 1995).

La representación gráfica de las distancias interraciales generadas por las diferencias en las frecuencias génicas y genotípica observadas se realizó mediante la confección de un cladograma a partir de las distancias genéticas de Nei (Nei 1987). Para el análisis antes señalado se utilizaron los programas Populations 1.2.28. (Langella 2002) ${ }^{5}$ y Treeview 1.6.6. (Page 2001).

http://www.bioinformatics.org/ tryphon/populations/ 6 http://taxonomy.zoology.gla.ac.uk/rod/rod.html

\section{RESULTADOS}

En el cuadro 2 se presentan las frecuencias génicas y genotípicas estimadas por raza y por locus. En el caso de la tiroglobulina, el polimorfismo de interés presenta una mayor frecuencia génica en las razas Angus Rojo y Angus Negro, con valores de 0,41 y 0,35 , respectivamente. Asociado a lo anterior y con respecto a lo que se observa en las otras razas, las frecuencias para el genotipo homocigoto TT, que favorece la infiltración de grasa intramuscular, también se encontraron más altas en dichos grupos raciales $(0,18$ y 0,11 , respectivamente). Por otra parte, las frecuencias más bajas para este polimorfismo se observaron en las razas Hereford y Clavel de Carne ( 0,10 y 0,17 , respectivamente).

El polimorfismo asociado al gen de leptina mostró una mayor frecuencia génica en las razas Hereford, Angus Negro y Angus Rojo (0,52, 0,48 y 0,48, respectivamente), mientras que las frecuencias génicas más bajas se observaron en las razas Bazadaise y Clavel de Carne ( 0,23 en ambos casos). En lo que respecta al genotipo TT, este presentó sus mayores frecuencias en las razas Simmental y Angus Negro $(0,28$ y 0,21 respectivamente). Prácticamente no se observaron individuos con dicho fenotipo en la raza Belga Azul.

El polimorfismo asociado a DGAT1 mostró una mayor frecuencia en la raza Bazadaise $(0,40)$, lo que coincidió con lo observado en las frecuencias del homocigoto (AA) que favorece el marmóreo. Las frecuencias génicas y genotípicas más bajas se advirtieron en Hereford y Belga Azul, donde no se observaron individuos con el genotipo AA.

El polimorfismo de interés asociado al gen FABP4 mostró mayores frecuencias en las razas Angus Rojo, Angus Negro y Simmental $(0,34,0,25$ y 0,25 , respectivamente) y la más baja en Belga Azul $(0,03)$. En lo que respecta al 
Cuadro 2. Frecuencias génicas y genotípicas estimadas por raza y por locus. El promedio muestra los genotipos y los alelos que predisponen a los animales a infiltrar grasa.

Gen and genotype frequencies estimated by breed and locus. The average shows the genotypes and alleles that predispose animals to marbling.

\begin{tabular}{|c|c|c|c|c|c|}
\hline \multirow{2}{*}{$\begin{array}{l}\text { Loci/Raza } \\
\text { Tiroglobulina }\end{array}$} & \multicolumn{3}{|c|}{ Frecuencias genotípicas } & \multicolumn{2}{|c|}{ Frecuencias génicas } \\
\hline & $\mathrm{TT}$ & $\mathrm{TC}$ & $\mathrm{CC}$ & $\mathrm{T}$ & $\mathrm{C}$ \\
\hline AN & 0,11 & 0,47 & 0,42 & 0,35 & 0,65 \\
\hline AR & 0,18 & 0,47 & 0,35 & 0,41 & 0,59 \\
\hline HF & 0,00 & 0,21 & 0,79 & 0,10 & 0,90 \\
\hline BA & 0,00 & 0,45 & 0,55 & 0,23 & 0,78 \\
\hline BD & 0,08 & 0,42 & 0,50 & 0,29 & 0,71 \\
\hline $\mathrm{CC}$ & 0,08 & 0,17 & 0,75 & 0,17 & 0,83 \\
\hline SI & 0,12 & 0,18 & 0,71 & 0,21 & 0,79 \\
\hline Promedio & $0,08 \pm 0,06$ & $0,34 \pm 0,14$ & $0,58 \pm 0,17$ & $0,25 \pm 0,11$ & $0,75 \pm 0,11$ \\
\hline Leptina & $\mathrm{TT}$ & $\mathrm{TC}$ & $\mathrm{CC}$ & $\mathrm{T}$ & $\mathrm{C}$ \\
\hline AN & 0,21 & 0,53 & 0,26 & 0,48 & 0,52 \\
\hline $\mathrm{AR}$ & 0,17 & 0,62 & 0,21 & 0,48 & 0,52 \\
\hline $\mathrm{HF}$ & 0,23 & 0,57 & 0,20 & 0,52 & 0,48 \\
\hline BA & 0,00 & 0,65 & 0,35 & 0,33 & 0,67 \\
\hline BD & 0,08 & 0,29 & 0,63 & 0,23 & 0,77 \\
\hline $\mathrm{CC}$ & 0,11 & 0,24 & 0,65 & 0,23 & 0,77 \\
\hline SI & 0,28 & 0,39 & 0,33 & 0,47 & 0,53 \\
\hline Promedio & $0,15 \pm 0,10$ & $0,47 \pm 0,16$ & $0,38 \pm 0,19$ & $0,39 \pm 0,13$ & $0,61 \pm 0,13$ \\
\hline DGAT1 & AA & $\mathrm{AK}$ & KK & A & $\mathrm{K}$ \\
\hline AN & 0,02 & 0,13 & 0,84 & 0,09 & 0,91 \\
\hline $\mathrm{AR}$ & 0,01 & 0,22 & 0,77 & 0,12 & 0,88 \\
\hline $\mathrm{HF}$ & 0,00 & 0,02 & 0,98 & 0,01 & 0,99 \\
\hline BA & 0,00 & 0,10 & 0,90 & 0,05 & 0,95 \\
\hline BD & 0,25 & 0,29 & 0,46 & 0,40 & 0,60 \\
\hline $\mathrm{CC}$ & 0,16 & 0,09 & 0,75 & 0,21 & 0,79 \\
\hline SI & 0,06 & 0,13 & 0,81 & 0,12 & 0,88 \\
\hline Promedio & $0,07 \pm 0,10$ & $0,14 \pm 0,09$ & $0,79 \pm 0,16$ & $0,14 \pm 0,13$ & $0,86 \pm 0,13$ \\
\hline FABP4 & GG & GC & $\mathrm{CC}$ & G & $\mathrm{C}$ \\
\hline AN & 0,06 & 0,37 & 0,57 & 0,25 & 0,75 \\
\hline $\mathrm{AR}$ & 0,16 & 0,36 & 0,48 & 0,34 & 0,66 \\
\hline $\mathrm{HF}$ & 0,01 & 0,22 & 0,77 & 0,12 & 0,88 \\
\hline BA & 0,00 & 0,06 & 0,94 & 0,03 & 0,97 \\
\hline BD & 0,00 & 0,42 & 0,58 & 0,21 & 0,79 \\
\hline $\mathrm{CC}$ & 0,06 & 0,29 & 0,65 & 0,21 & 0,79 \\
\hline SI & 0,00 & 0,50 & 0,50 & 0,25 & 0,75 \\
\hline Promedio & $0,04 \pm 0,06$ & $0,32 \pm 0,14$ & $0,64 \pm 0,16$ & $0,20 \pm 0,10$ & $0,80 \pm 0,10$ \\
\hline
\end{tabular}

El promedio señala las frecuencias del genotipo y el alelo que predispone a infiltrar una mayor cantidad de grasa. Se indica el promedio de las frecuencias por locus más su desviación estándar. 
Cuadro 3. Heterocigosis observada y esperada, índice de consanguinidad para cada una de las razas, loci evaluados y prueba de equilibrio de Hardy y Weinberg.

Observed and expected heterozygosity, inbreeding rate for each breed and locus and Hardy \& Weinberg equilibrium test.

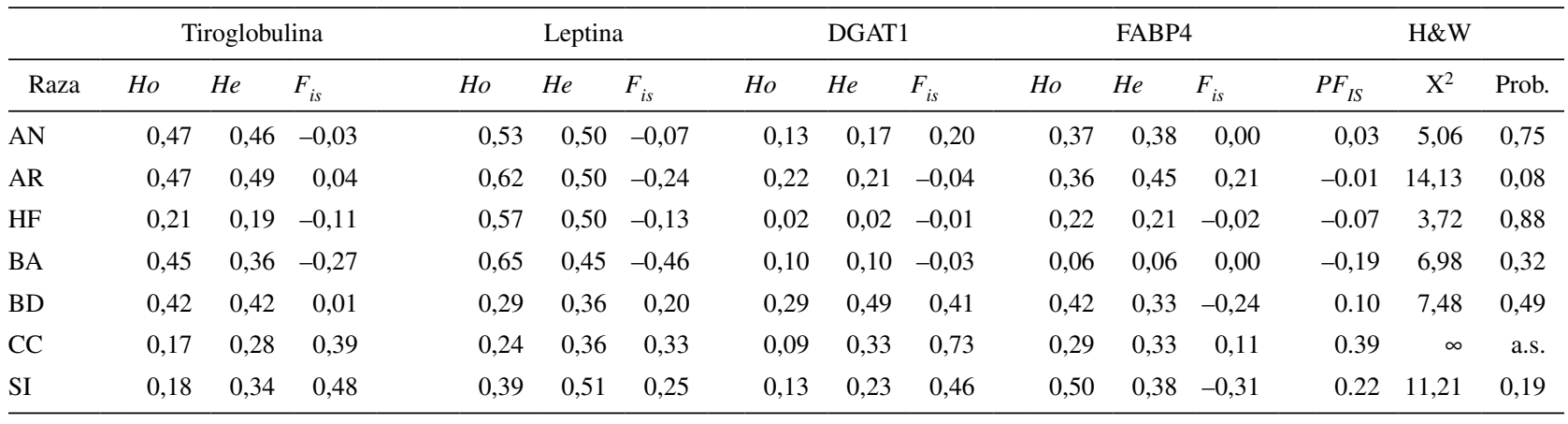

$\mathrm{Ho}$, heterocigosis observada.

$\mathrm{He}$, heterocigosis esperada.

$F_{i s}$, coeficiente de consanguinidad.

$H \& W$, Prueba de equilibrio de Hardy y Weinberg por poblaciones $(\mathrm{P}<0,05)$
$P F_{I S}$, FIS promedio

$\mathrm{X}^{2}$, chi-cuadrado.

Prob., probabilidad estimada por raza.

$\infty$, probabilidad muy elevada, tendiente a infinito a.s., altamente significativo. genotipo GG, este mostró una frecuencia relativamente más alta en la raza Angus Rojo $(0,16)$.

Al comparar las frecuencias observadas con las frecuencias esperadas se pudo observar que la mayoría de las razas evaluadas presentan distribuciones de frecuencias compatibles con el equilibrio de Hardy y Weinberg. En este contexto, si se contrastan los valores de la heterocigosis observada $(\mathrm{Ho})$, heterocigosis esperada $(\mathrm{He})$ y los valores para el estadístico $F_{I S}$, se puede advertir que las diferencias entre ambas variables resultan muy bajas, y con valores de $F_{I S}$ muy próximos a cero (cuadro 3 ). La excepción se observa en la raza Clavel de Carne, la cual presenta diferencias altamente significativas.

La figura 1 muestra el cladograma confeccionado a partir de las distancias genéticas de Nei. En este se aprecia la conformación de tres clusters claramente definidos. En el primero se encuentra la raza Simmental, en el segundo, las razas Hereford, Belga Azul, Bazadaise y Clavel de Carne, mientras que en el tercero se agrupan las razas Angus Rojo y Angus Negro.

\section{DISCUSIÓN}

En términos generales se observa que la distribución de las frecuencias de los polimorfismos en dos de los genes evaluados mostraron valores cercanos a los observados por otros autores que han estudiado dichas variantes en algunas de las razas incluidas en el presente trabajo (Buchanan y col 2002, Kaupe y col 2004, Casas y col 2005, Casas y col 2007, Barrendse y col 2009 y Ayres y col 2010). De esta manera, se observó que en el gen leptina la frecuencia más alta para el alelo $\mathrm{T}$ que predispone a infiltrar grasa, se encuentra en las razas Hereford, Angus Negro y Angus Rojo. Un resultado muy parecido obtuvieron Buchanan y col (2002) quienes evaluaron el mismo polimorfismo en varias razas bovinas de carne, encontrando que las mayores frecuencias para este alelo se encontraban precisamente en estas razas. Resultados similares se obtuvieron en el gen DGAT1, donde el alelo que predispone a infiltrar grasa (A) mostró una menor frecuencia en las razas Belga Azul y Hereford. Dicho resultado había sido informado por Kaupe y col (2004), quienes encontraron una frecuencia

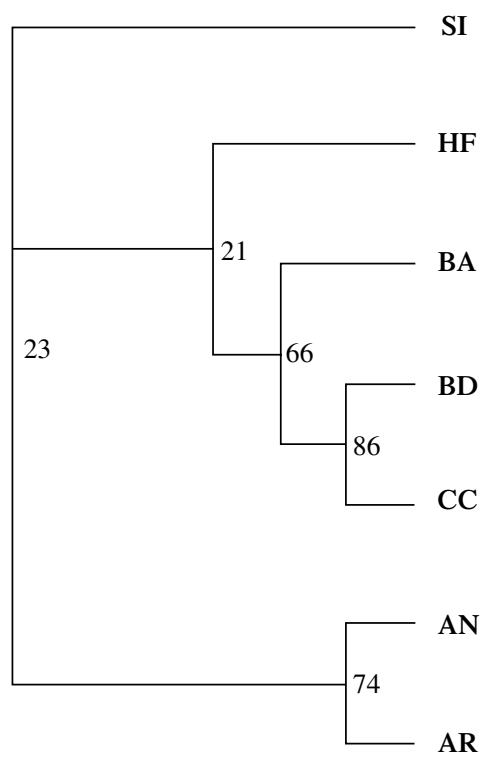

Figura 1. Cladograma confeccionado a partir de las distancias genéticas de Nei. Aunque la gráfica no resulta representativa de las verdaderas distancias genéticas existentes entre las razas, en ella pueden apreciarse los agrupamientos generados por las diferencias en las frecuencias de los polimorfismos estudiados.

Nei genetic distances cladogram. Although the graph is not representative of the true genetic distances between breeds, the clusters, generated by differences in frequencies of the polymorphisms studied, can be clearly observed. 
muy baja de dicho polimorfismo en las razas anteriormente señaladas. En lo que respecta a los polimorfismos estudiados en los genes Tiroglobulina y FABP4, en estos no se observaron frecuencias parecidas a las descritas en la literatura, siendo estas incluso bastante inferiores a las observadas por autores como Casas y col $(2005,2007)$, Barrendse y col (2009) y Ayres y col (2010).

En general, los resultados del presente trabajo ponen en evidencia los efectos directos e indirectos generados por los procesos de selección de los alelos relacionados con infiltración grasa sobre los distintos grupos raciales evaluados. En un extremo se encuentra la raza británica Aberdeen Angus representada por Angus Rojo y Angus Negro, que mostró frecuencias elevadas de alelos favorables a infiltración grasa en tres de los cuatro loci analizados, respecto de las otras razas. Cabe señalar que, a nivel fenotípico, este grupo de animales se caracteriza por mostrar altos niveles de infiltración grasa en comparación con otras razas de origen continental, que generalmente han sido seleccionadas para producir carnes magras. En el otro extremo se encuentran las razas Belga Azul y Hereford con frecuencias bajas respecto a lo observado en los otros grupos raciales. Este resultado era particularmente esperable en Belga Azul, que al igual que otras razas no incluidas en el presente estudio ha sido seleccionada en base a una serie de mutaciones que favorecen la formación de hipertrofia muscular, también conocida como doble pulpa, la cual se caracteriza por un bajo contenido de infiltración grasa en la carne (Casas y col 2000). De esta manera, los animales de dicha raza suelen presentar altos rendimientos debido a sus grandes masas musculares ubicadas principalmente en el cuarto trasero, característica que ha ido en desmedro de la infiltración grasa.

Un caso distinto es el observado en la raza Hereford, que al ser de origen británico se esperaba presentara frecuencias más elevadas en los alelos que favorecen la infiltración grasa, tal y como lo han informado autores como Buchanan (2002). Sin embargo, al igual que lo observado por Kaupe y col (2004), en nuestro estudio las frecuencias de los alelos que favorecen el marmóreo fueron más bien bajas. El resultado antes señalado podría atribuirse a que el $90 \%$ de los animales muestreados para el presente estudio provenían de un único predio, lo que no permite descartar algún efecto asociado al sistema de selección empleado en este predio, el cual podría dar lugar a una presión de selección hacia los genotipos con baja predisposición a infiltrar grasa.

El cladograma presentado en la figura 1 ratifica el planteamiento de que la presión selectiva ejercida por años sobre ciertas razas de carne ha afectado indirectamente la distribución de sus frecuencias alélicas. De esta manera, aunque dicha imagen no representa las distancias genéticas reales entre las razas evaluadas, es posible advertir un claro agrupamiento de la raza Aberdeen Angus (AR y AN), que presentó frecuencias altas en tres de los cuatro polimorfismos evaluados.
En lo que respecta a la distribución de las frecuencias genotípicas, sorprende que seis (86\%) de los grupos raciales se encuentren en equilibrio de Hardy y Weinberg para los marcadores evaluados. Dicho resultado es interesante al constatar que Johnston y Graser (2010) observaron que al evaluar 12 marcadores moleculares incluidos en la prueba comercial GeneStart, en diferentes razas bovinas de carne utilizadas en Australia, el 86\% de estas se encontraban en equilibrio de Hardy y Weinberg.

Conforme lo anterior, vale la pena señalar que dicho resultado no era esperado en el presente estudio ya que los rebaños de especies domésticas suelen ser considerados poblaciones pequeñas, generalmente expuestas a procesos dispersivos, los cuales pueden cambiar aleatoriamente sus frecuencias génicas (deriva génica) y por ende sus frecuencias genotípicas (Falconer y MacKay 1996). De acuerdo con esto, los resultados observados en el presente trabajo podrían ser consecuencia del azar y de las fluctuaciones aleatorias en las frecuencias intergeneracionales producto del muestreo gamético (Falconer y MacKay 1996). A pesar de esto, existen algunos aspectos que no concuerdan con lo esperado en poblaciones pequeñas sometidas a efectos dispersivos. Por ejemplo, si los planteles evaluados se encontrasen bajo dichos efectos, se esperaría observar una menor variabilidad genética, expresada en bajos niveles de heterocigosis. Muy por el contrario, los valores de heterocigosis observada en los marcadores evaluados alcanzan cifras bastante próximas a lo esperado en poblaciones grandes donde no existe selección, mutación ni migración, y en las que las frecuencias génicas y genotípicas permanecen inalterables de generación en generación (Falconer y Mackay 1996). Por otro lado, si las poblaciones se encontraran sufriendo los efectos de la deriva genética, es decir, sus frecuencias génicas y genotípicas cambiaran aleatoriamente de generación en generación, sería muy poco probable que los resultados coincidiesen con lo obtenido por otros autores en otros países y en las mismas razas (Buchanan y col 2002, Kaupe y col 2004, Barendse y col 2009).

Un aspecto interesante a tener en cuenta es que el único grupo racial que presentó diferencias altamente significativas entre las frecuencias genotípicas observadas y esperadas fue Clavel de Carne, raza chilena originada a partir de cruzas entre Overo Colorado y Shorton y que comenzó a ser seleccionada hace alrededor de 40 años. La principal diferencia de manejo reproductivo entre dicha raza y el resto de las razas incluidas en el presente estudio radica en que la mayoría de los productores realizan el servicio de sus hembras mediante cubierta natural o inseminación con semen proveniente de toros locales, ubicados en los predios pertenecientes a la asociación de criadores de la raza. Esta práctica, que tiene como objetivo mantener la pureza de los animales, podría estar generando efectos contraproducentes debido al reducido número de toros utilizados como reproductores y a la escasez de registros 
genealógicos confiables necesarios para controlar problemas de endogamia.

Es posible entonces plantear que los equilibrios observados en la mayoría de las otras razas podrían estar asociados a factores como el uso de semen importado, a las cruzas con otras razas y al manejo intrapredial, los cuales podrían provocar que el comportamiento de los planteles evaluados no sea el de una población pequeña sometida a efectos dispersantes, sino más bien el de una población grande con frecuencias inherentemente estables. Para verificar esta hipótesis se requeriría al menos de la implementación de registros genealógicos adecuados y la evaluación de varias generaciones de animales.

Un importante aspecto que debe ser discutido a partir de los resultados anteriormente presentados, dice relación con la factibilidad de utilizar estos marcadores moleculares en programas de mejoramiento genético. En este aspecto, es importante tener en cuenta que existe evidencia que señala que la excesiva e inadecuada presión selectiva generada sobre un número limitado de genes puede llegar a producir altos niveles de endogamia (Gibson 1994). Este problema ha sido analizado y discutido por diversos autores que además han agregado que el uso inadecuado de dichas herramientas podría provocar que a largo plazo se generen importantes reducciones de la respuesta a la selección (Gibson 1994). Por esta razón, se ha puesto énfasis en la conveniencia de incorporar la información de los marcadores genéticos en los modelos matemáticos (BLUP) utilizados en la valoración genética de reproductores. En este contexto, investigadores de cuatro universidades de los EE.UU. (Cornell, Iowa, Colorado y Georgia) conformaron en julio de 2001 el NBCEC, con el objetivo principal de integrar la información entregada por los marcadores moleculares a los modelos mixtos (maBLUP) (Agric 2006). De igual manera, autores como Li y col (2008) han desarrollado estrategias de selección asistida por marcadores moleculares, destinadas a maximizar la respuesta genética a largo plazo, llegando a controlar de manera muy eficiente la variabilidad genética y los niveles de endogamia existentes en los rebaños.

En términos generales y a modo de conclusión, es posible plantear que los marcadores moleculares evaluados en el presente estudio presentan frecuencias parecidas a las que se han observado en otros estudios, y que su uso en programas de mejoramiento genético asistido por marcadores moleculares es factible. No obstante lo anterior, dado que estos y otros marcadores moleculares han sido probados en el extranjero, en razas, ambientes y condiciones de crianza distintos a los existentes en Chile, se hace imprescindible que entidades independientes como las universidades y centros de investigación nacionales continúen con la realización de estudios a nivel local, destinados a la validación de dichos marcadores. Esta necesidad ha sido confirmada por distintas universidades y organismos como el NBCEC y el Centro de Investigación Cooperativa de Carne Bovina de Australia (CRC, del inglés Beef Cooperative Research Centre), los cuales han informado sobre la detección de inconsistencias en los valores genéticos determinados mediante el uso de marcadores moleculares incorporados en las pruebas comerciales (Rincker y col 2006, Quaas y col 2006, Van Eenennaam y col 2007, Dikeman y col 2007, Casas y col 2007, Johnston y Graser 2010).

De igual manera, y tal y como ha sido corroborado por autores como Dikeman y col (2007) y Johnston y Graser (2010), dado que la distribución de las frecuencias de los polimorfismos asociados a caracteres de interés productivo no suelen ser similares en todos los grupos raciales, es necesario que la aplicación de este tipo de tecnología cuente con información genealógica confiable (registros), de manera de evitar el surgimiento de problemas derivados de la depresión endogámica. Por último, es necesario que los productores y asociaciones de productores se asesoren por profesionales u organismos técnicos que cuenten con las competencias necesarias para integrar la información genotípica entregada por los marcadores moleculares y los métodos de selección genético-cuantitativa tradicionales como el modelo animal, de manera de poder corregir los efectos de la componente ambiental, estimar los progresos genéticos de manera adecuada y evitar eventuales pérdidas de respuesta a la selección.

\section{RESUMEN}

La elección de los reproductores es un paso fundamental en todo programa de mejora genética, por esta razón la metodología para la evaluación de los animales ha evolucionado rápidamente, y en las últimas décadas ha incorporado el uso de marcadores moleculares. Varias empresas trasnacionales han puesto a disposición de los productores pruebas comerciales basados en estas tecnologías, las cuales permiten evaluar la predisposición genética de los animales a manifestar determinado fenotipo. A pesar de lo anterior, han comenzado a surgir dudas sobre los efectos negativos que podrían generarse en los rebaños por el uso inadecuado de marcadores moleculares, específicamente problemas asociados a la depresión endogámica y a la pérdida a largo plazo, de respuesta a la selección. Conforme lo anterior, el objetivo del presente trabajo fue determinar las frecuencias génicas y genotípicas de cuatro polimorfismos asociados a infiltración grasa intramuscular, en 7 razas bovinas utilizadas para la producción de carne en la Región de La Araucanía. Se evaluaron los genes de Leptina, Tiroglobulina, DGAT1 y FABP4, los cuales presentaron distribuciones de frecuencias que variaron bastante entre razas, observándose las mayores frecuencias de alelos favorables a infiltración en la raza Aberdeen Angus, y las menores en Belga Azul y Hereford. Se concluye que es factible la utilización de dichas herramientas en programas de mejoramiento genético, pero que es necesario contar con registros genealógicos confiables y con el asesoramiento de profesionales u organismos técnicos que cuenten con las competencias necesarias para integrar la información genotípica proveniente de los marcadores moleculares a los modelos utilizados para la selección de reproductores.

\section{AGRADECIMIENTOS}

Agradecemos la colaboración de los señores Claudio Crovetto, Héctor Rodríguez, José Miguel García, Mario García, Leonardo García, Hernán Montenegro, Patricio Bornard y Jimmie Dungan, importantes productores ganaderos de la Región de La Araucanía, sin los cuales no habría sido posible la ejecución del presente estudio. 


\section{REFERENCIAS}

Agric B. 2006. An economic analysis of gene marker assisted seedstock selection in beef cattle. Master of Science, University of British Columbia, Canada.

Ayres DR, FRP Souza, MEZ Mercadante, LFS Fonseca, H Tonhati, JNSG Cyrillo, SFM Bonilha, LG Albuquerque. 2010. Evaluation of TFAM and FABP4 gene polymorphisms in three lines of Nellore cattle selected for growth. Gene Mol Res 9, 2050-2059.

Barendse W. 1999. Assessing lipid metabolism. Int. Pat. Appl. PCT/ AU98/00882, Int Pat Publ WO 99/23248.

Barendse W, RJ Bunch, MB Thomas, BE Harrison. 2009. A splice site single nucleotide polymorphism of the fatty acid binding protein 4 gene appears to be associated with intramuscular fat deposition in longissimus muscle in Australian cattle. Anim Gene 40, 770-773.

Barton-Gade PA. 1988. The effect of breed on meat quality characteristics in pigs. In: Proceedings $34^{\text {th }}$ International Congress of Meat Science and Technology, Brisbane, Australia, Pp 56-570.

Belkhir K, P Borsa, L Chikhi, N Raufaste, F Bonhomme. 2002. GENETIX 4.04, logiciel sous Windows TM pour la génétique des populations. Laboratoire Genome, Populations, Interactions, CNRS UMR 5000, Université de Montpellier II, Montpellier, France.

Block SS, WR Butler, RA Ehrhardt, AW Bell, ME Van Amburgh, YR Boisclair. 2001. Decreased concentration of plasma leptin in periparturient dairy cows is caused by negative energy balance. J Endocrinol 171, 339-348.

Buchanan FC, CJ Fitzsimmons, AG Van, TD Thue, DC WindkelmanSim, SM Schmutz. 2002. Association of a missense mutation in the bovine leptin gene with carcass fat content and leptin mRNA levels. Gen Sel Evol 34, 105-116.

Buhman KF, HC Chen, RV Farese Jr. 2001. The enzymes of neutral lipid synthesis. J Biol Chem 276, 40369-40372.

Buxade C. 1995. Zootecnia, Bases de producción animal. Tomo IV. Ediciones Mundi-Prenza, Madrid, España, Pp 153-180.

Casas E, SD Shackelford, JW Keele, RT Stone, SM Kappes, M Koohmaraie. 2000. QTL affecting growth and carcass composition of cattle segregating alternate forms of myostatin. J Anim Sci 78, 560-569.

Casas E, SN White, DG Riley, TPL Smith, RA Brenneman, TA Olson, DD Johnson, SW Coleman, GL Bennett, CC Chase Jr. 2005. Assessment of single nucleotide polymorphisms in genes residing on chromosomes 14 and 29 for association with carcass composition traits in Bos indicus cattle. J Anim Sci 83, 13-19.

Casas E, SN White, SD Shackelford, TL Wheeler, M Koohmaraie, GL Bennett, TP Smith. 2007. Assessing the association of single nucleotide polymorphisms at the thyroglobulin gene with carcass traits in beef cattle. J Anim Sci 85, 2807-2814.

CORPOICA. 2005. Patrones tecnológicos y calidad de la carne bovina en el caribe colombiano. Editorial Produmedios, Bogotá, Colombia, Pp 75-82.

Corva PM, GV Fernández-Macedo, LA Soria, J Papaleo-Mazzucco, M Motter, EL Villarreal, A Schor, CA Mezzadra, LM Melucci, MC Miquel. 2009. Effect of leptin gene polymorphisms on growth, slaughter and meat quality traits of grazing Brangus steers. Gen Mol Res 8, 105-116.

Dikeman ME, AL Van Eenennaam, J Li, RM Thallman, RL Quaas, C Gill, DE Franke, MG Thomas. 2007. Validation of commercial DNA tests for beef quality traits. Día de Campo Universidad del Estado de Kansas, Manhattan, NY, USA, Pp 36-42.

Falconer DS, TFC MacKay. 1996. Introduction to Quantitative Genetics. $4^{\text {th }}$ ed. Longman Scientific and Technical, New York, USA, Pp 49-83.

Fisher RA. 1935. The logic of inductive inference (with discussion). $J$ R Stat Soc 98, 39-82.

Fitzsimmons CJ, SM Schmutz, RD Bergen, JJ McKinnon. 1998. A potential association between the BM 1500 microsatellite and fat deposition in beef cattle. Mamm Genome 9, 432-434.

Gibson, JP 1994. Short-term gain at the expense of long-term response with selection of identified loci. Proceedings of the $5^{\text {th }}$ World
Congress on Genetics Applied to Livestock Production, Guelph, Canada 21, 201-204.

Goudet J. 1995. FSTAT (Ver. 2.9.3.2): a computer program to calculate F-statistics. J Heredity, 86, 485-486.

Grisart B, W Coppieters, F Farnir, L Karim, C Ford, P Berzi, N Cambisano, M Mni, S Reid, P Sirnon, R Spelman, M Georges, R Snell. 2002. Positional candidate cloning of a QTL in dairy cattle: Identification of a missense mutation in the bovine DGAT1 gene with major effect on milk yield and composition. Genome Res 12, 222-231.

Grisart B, F Farnir, L Karim, N Cambisano, JJ Kim, A Kvasz, M Mni, P Simon, JM Frere, W Coppieters, M Georges. 2004. Genetic and functional confirmation of the causality of the DGAT1 K232A quantitative trait nucleotide in affecting milk yield and composition. Proceedings of the National Academy of Sciences of USA 101, 2398-2403.

Harper GS, DW Pethick. 2001. The physiology of marbling: what is it, and why does it develop? Marbling Symposium: Proceedings of CRC Conference, Coffs Harbour, Australia, Pp 36-45.

Hazel LN. 1943. The Genetic Basis for Constructing Selection Indexes. Genetics 28, 476-490.

Henderson CR. 1949. Estimates of changes in herd environment. J Dairy Sci 32, 706.

Henderson CR. 1990. Statistical methods in animal improvement: historical overview. In: Gianola D, Hammond K (eds). Statistical methods for genetic improvement in livestock. Springer-Verlag, Berlin, Germany, Pp 2-14.

Jiang Z, JJ Michal. 2007. Polymorphisms in Fatty Acid Binding Protein 4 ("FABP4") gene and their associations with measures of marbling and subcutaneous fat depth beef cattle. US Pat $\mathrm{N}^{\circ}$ US2007/0020658 A1.

Johnston DJ, HU Graser. 2010. Estimated gene frequencies of GeneSTAR markers and their size of effects on meat tenderness, marbling, and feed efficiency in temperate and tropical beef cattle breeds across a range of production systems. J Anim Sci 88,1917-1935

Kadokawa H, D Blache, Y Yamada, GB Martin. 2000. Relationships between changes in plasma concentrations of leptin before and after parturition and the timing of first post-partum ovulation in high-producing Holstein dairy cows. Reprod Fertil Dev 12, 405-411.

Kaikaus RM, NM Bass, RK Ockner. 1990. Functions of fatty acid binding proteins. Experientia 46, 617-630.

Kaupe B, A Winter, R Fries, G Erhardt. 2004. DGAT1 polymorphism in Bos indicus and Bos taurus cattle breeds. J Dairy Res 71, 182-187.

Kononoff PJ, HM Deobald, EL Stewart, AD Laycock, FLS Marquess. 2005. The effect of a leptin single nucleotide polymorphism on quality grade, yield grade, and carcass weight of beef cattle. $J$ Anim Sci 83, 927-932.

Li Y, H Kadarmideen, J Dekkers. 2008. Selection on multiple QTL with control of gene diversity and inbreeding for long-term benefit. Ann Breed Gen 125, 320-329.

Michal JJ, ZW Zhang, CT Gaskins, Z Jiang. 2006. The bovine fatty acid binding protein 4 gene is significantly associated with marbling and subcutaneous fat depth in Wagyu x Limousin F2 crosses. Anim Gen 37, 400-402.

Nei M. 1987. Molecular Evolutionary Genetics. Columbia University Press, Nueva York, USA.

Powell RL, HD Norman. 2006. Major Advances in Genetic Evaluation Techniques. J Dairy Sci 89, 1337-1348.

Quaas RL, J Li, RM Thallman, AL Van Eenennaam, RL Fernando, C Gill. 2006. Validation of commercial DNA tests for quantitative beef traits. $8^{\text {th }}$ World Congress on Genetics Applied to Livestock Production. Belo Horizonte, MG, Brasil, Pp 13-18.

Raymond M, F Rousset. 1995. GENEPOP, Version 3.3: population for exact test ecumenism. J Heredity 86, 248-249

Rincker CB, NA Pyatt, LL Berger, DB Faulkner. 2006. Relationship among GeneSTAR marbling marker, intramuscular fat deposition, and expected progeny differences in early weaned Simmental steers. J Anim Sci 84, 686-693. 
Riquet J, W Coppieters, N Cambisano. 1999. Fine-mapping of quantitative trait loci by identity by descent in out bred populations: application to milk production in dairy cattle. Proc Natl Acad Sci USA, 96, 9252-9257.

Robertson A, WG Hill. 1984. Deviations from Hardy-Weinberg proportions: sampling variances and use in estimation of inbreeding coefficients. Genetics 107, 703-718.

Santos-Alvarez J, R Goberna, V Sánchez-Margalet. 1999. Human leptin stimulates proliferation and activation of human circulating monocytes. Cell Immunol 194, 6-11.

Shanks RD. 2008. A revolution in dairy cattle genetics. IL Dairy Day Report, Pp 37-38.

Smith HF. 1936. A discriminant function for plant selection. Ann Eugenics 7, 240-250

Spelman RJ, CA Ford, P McElhinney, GC Gregory, RG Snell. 2002. Characterization of the DGAT1 gene in the New Zealand dairy population. J Dairy Sci 85, 3514-3517.

Stone RT, SM Kappes, C Beattie. 1996. Two polymorphic microsatellites within an $18 \mathrm{~kb}$ genomic clone containing the bovine ob gene. Anim Genetics 27(Suppl. 2), 64.
Thaller G, W Kramer, A Winter, B Kaupe, G Erhardt, R Fries. 2003ª Effects of DGAT1 variants on milk production traits in German cattle breeds. J Anim Sci 81, 1911-1918.

Thaller G, C Kuhn, A Winter, G Ewald, O Bellmann, J Wegner, H Zuhlke, R Fries. 2003 ${ }^{\text {b }}$ DGAT1, a new positional and functional candidate gene for intramuscular fat deposition in cattle. Anim Genet 34, 354-357.

Van Eenennaam AL, J Li, RM Thallman, RL Quaas, ME Dikeman, CA Gill, DE Franke, MG Thomas. 2007. Validation of commercial DNA tests for quantitative beef quality traits. J Anim Sci 85, 891-900.

Weir BS, CC Cockerham. 1984. Estimating F-statistics for the analysis of population structure. Evolution 38, 1358-1370.

Winter A, W Kramer, FA Werner, S Kollers, S Kata, G Durstewitz, J Buitkamp, JE Womack, G Thaller, R Fries. 2002. Association of a lysine-232/alanine polymorphism in a bovine gene encoding acyl-CoA: diacylglycerol acyltransferase (DGAT1) with variation at a quantitative trait locus for milk fat content. Proceedings of the National Academy of Sciences of USA 99, 9300-9305.

Wright S. 1965. The interpretation of population structure by F-statistics with special regard to systems of mating. Evolution 19, 395-420. 